\title{
A Preliminary Study of Peer-to-Peer Human-Robot Interaction
}

\author{
Terrence Fong, Jean Scholtz, Julie A. Shah, Lorenzo Flückiger, \\ Clayton Kunz, David Lees, John Schreiner, Michael Siegel, Laura M. Hiatt, \\ Illah Nourbakhsh, Reid Simmons, Robert Ambrose, Robert Burridge, \\ Brian Antonishek, Magda Bugajska, Alan Schultz and J. Gregory Trafton
}

\begin{abstract}
The Peer-to-Peer Human-Robot Interaction (P2PHRI) project is developing techniques to improve task coordination and collaboration between human and robot partners. Our work is motivated by the need to develop effective human-robot teams for space mission operations. A central element of our approach is creating dialogue and interaction tools that enable humans and robots to flexibly support one another. In order to understand how this approach can influence task performance, we recently conducted a series of tests simulating a lunar construction task with a human-robot team. In this paper, we describe the tests performed, discuss our initial results, and analyze the effect of intervention on task performance.
\end{abstract}

\section{INTRODUCTION}

\section{A. The Peer-to-Peer Human-Robot Interaction Project}

Since February 2004, NASA has been pursuing the "Vision for Space Exploration" (VSE) [1]. The primary goal of the VSE is to "implement a sustained and affordable human and robotic program to explore the solar system and beyond", beginning with robotic missions to the Moon in 2008 and leading eventually to human exploration of Mars. To achieve this goal, humans and robots will need to collaborate to perform basic mission tasks including structural assembly, inspection, and maintenance.

The objective of the "Peer-to-Peer Human-Robot Interaction" (P2P-HRI) project, therefore, is to develop HRI techniques that allow humans and robots to work effectively together [2]. We use the term "peer-to-peer" not because we expect humans and robots to have equal capabilities, but to emphasize the idea that humans and robots should work as partners, i.e., that their roles should be as balanced as possible. In particular, we believe that it is possible to compensate for failures and limitations of autonomy through interaction and teamwork.

Our research focuses on making human-robot teams more effective for space exploration by: (1) developing natural interaction mechanisms; (2) reducing the workload associated with robot control; and (3) maximizing the work that the

T. Fong, L. Flückiger, C. Kunz, D. Lees, J. Schreiner, and M. Siegel are with the NASA Ames Research Center, Moffett Field, CA 94035

L. Hiatt, I. Nourbakhsh, and R. Simmons are with the Robotics Institute, Carnegie Mellon University, Pittsburgh, PA 15213

R. Ambrose and R. Burridge are with the NASA Johnson Space Center, Houston, TX 77058

J. Scholtz and B. Antonishek are with the National Institute of Standards and Technology, Gaithersburg, MD 20899

M. Bugajska, A. Schultz and J.G. Trafton are with the Naval Research Laboratory, Washington, DC 20375

J. Shah is with the Massachusetts Institute of Technology, Cambridge, MA 02139 humans and robots can accomplish together. In addition, we are developing methods that enable human-robot teams to operate independently of ground control. This capability is needed when communications are intermittent (e.g., exploration in deep craters) or significantly time-delayed.

There are three primary components in our approach. First, we are developing an interaction framework called the "Human-Robot Interaction Operating System" (HRI/OS). The HRI/OS is designed to enable humans and robots to engage in task-oriented dialogue and problem solving. Second, we are using computational cognitive architectures to model human behavior and make human and robot more understandable to each other. Finally, we are developing evaluation procedures using human-robot teams, analog environments, and quantitative HRI metrics [3].

\section{B. Human-Robot Interaction Operating System}

In order for humans and robots to work effectively together, they need to be able to converse about abilities, goals and achievements. The Human-Robot Interaction Operating System (HRI/OS) is an agent-based system that provides coordination and interaction services for human-robot teams [4]. A key feature of the HRI/OS is that it allows robots to ask questions of humans, so that robots can obtain human assistance for cognition and perception tasks.

We designed the HRI/OS to support the performance of operational tasks. In space exploration, operational tasks include: construction, structural inspection and maintenance, and in-situ resource collection and transport [5]. The current version of the HRI/OS is implemented using the Open Agent Architecture (OAA) [6] and the Internet Communication Engine (ICE) middleware [7].

The primary components of the HRI/OS (Figure 1) are:

1) Task Manager: The Task Manager (TM) is responsible for coordinating and managing the execution of operational tasks. It does this by decomposing the overall goal of the system into high-level tasks, which are assigned to humans or robots for execution. Only a single task is assigned to a given agent at a time. Unlike traditional executives, the TM does not know anything about low-level task details. Instead, it relies on each agent to work in a distributed, independent manner, managing and monitoring their own task execution.

2) Resource Manager: The Resource Manager (RM) is responsible for continually tracking which resources (human, robots, services) are available and what capabilities each resource possesses. When an agent has a request, such as 


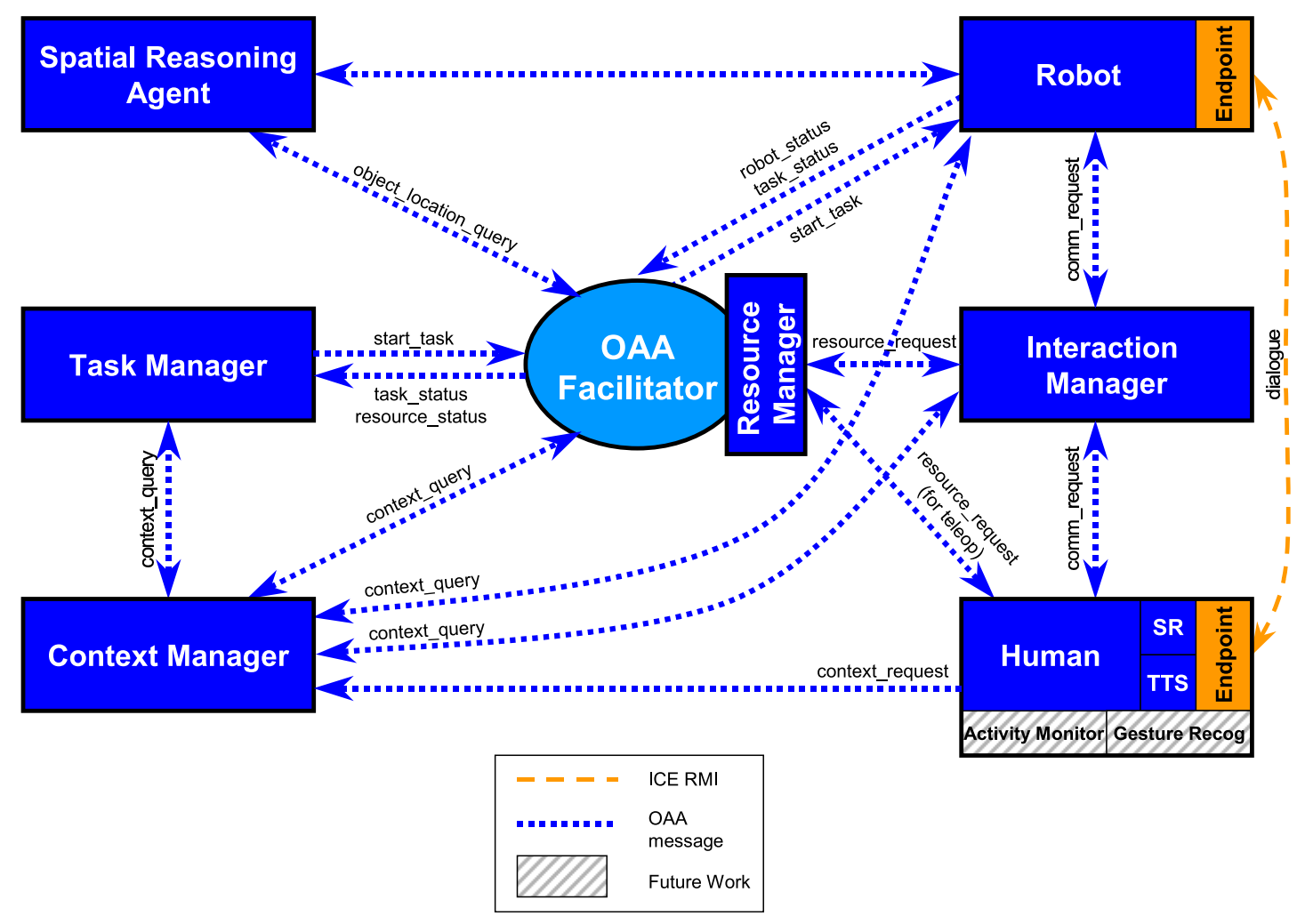

Fig. 1. The Human-Robot Interaction Operating System (HRI/OS) is an agent-based system.

a task to be performed or a question to be answered, the RM generates a prioritized list of agents to be consulted. Unlike facilitation in most agent systems, the RM performs delegation using multiple criteria that can vary with time and situation, rather than simple match making. In particular, in addition to service matching, the RM considers a variety of factors including availability, physical location, workload, past performance, etc.

3) Interaction Manager: The Interaction Manager (IM) coordinates dialogue between agents. The IM gives each agent the ability to communicate with other agents: to ask for help, to respond to questions, etc. In the HRI/OS, agents communicate with one another via point-to-point "dialogue endpoints". Endpoints allow agents to send a variety of data (text, images, sensor readings, etc.) to each other. The HRI/OS currently includes graphical and speech user interfaces. Other interface modalities (e.g., visual gesturing) will be included in the future.

4) Context Manager: In a complex agent system, keeping track of the activities and state of agents over time is a difficult task. This is particularly true when multiple agents operate in parallel and when activity is observed remotely (e.g., via user interfaces). Thus, to facilitate situational awareness, we have developed a Context Manager (CM). The CM keeps track of everything that occurs while the system is running: task status and execution, agent activities, agent dialogue, etc. Then, when agents have need to recall history, they can query the CM for a summary of information.
5) Spatial Reasoning Agent: When human-robot teams perform operational tasks, such as construction, understanding and communicating spatial dialogue plays a significant role [8]-[10]. In particular, when humans and robots work in a shared environment, robots must be able to understand how humans perceives space and the relative positions of objects around them. Thus, the HRI/OS includes a spatial reasoning agent (SRA), which resolves spatial ambiguities in dialogue (e.g., "move the light to the left of the box"). The current implementation of the SRA is capable of resolving frame of reference ambiguities including ego-, addresse-, object-, and exo-centric references using a computational cognitive model [11]-[13].

\section{INITIAL Evaluation}

\section{A. Use Case Study}

In November 2005, to assess our progress in developing with peer-to-peer HRI, we conducted a study of a simulated construction project involving seam welding and inspection by a human-robot team. Seam welding is a task that will be required for building and maintaining a variety of structures on planetary surfaces [14]. For example, linear welds might be used to construct pressure vessels, work hangers, and shelters too large to raise into space in one piece [15].

The human-robot team consisted of five different members working in parallel (Figure 2). In each trial, the team worked to retrieve panels from a storage depot, place the panels on a structural frame, weld adjacent panels, and inspect the welds. Two people wearing spacesuits performed the roles 


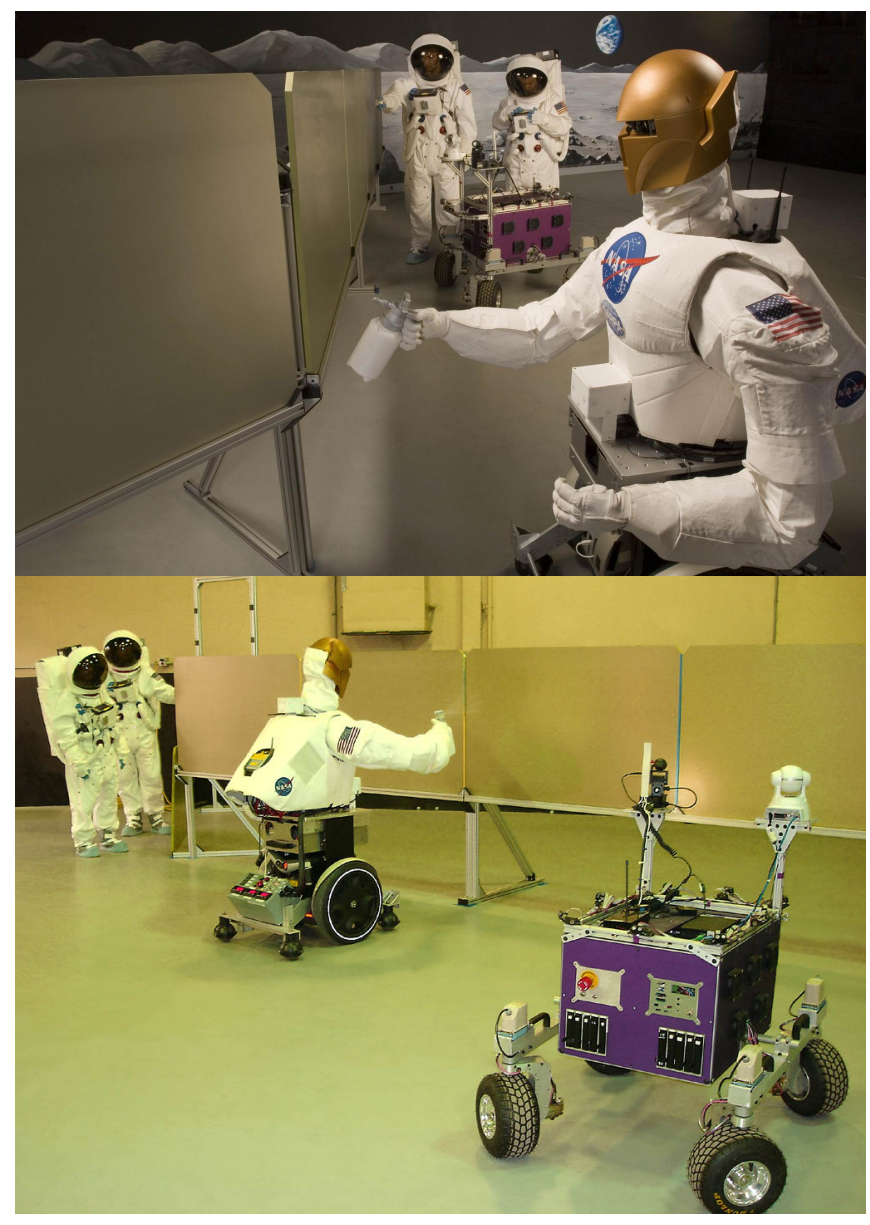

Fig. 2. Human-robot team working on a simulated construction project. During the study, two astronauts placed panels on a frame, the Robonaut humanoid robot created seam welds, and the K10 mobile robot inspected the welds. A third person (not shown) remotely supported the team from inside a habitat mock-up.

of astronauts in $\mathrm{EVA}^{1}$, retrieving and placing the panels. The Robonaut humanoid robot [16] from Johnson Space Center worked as a welder using a simulated seam weld process. The K10 rover from Ames Research Center was responsible for inspecting the welded seams. An additional person, IVA ${ }^{2}$, remotely supported the construction project from inside a habitat mockup.

This work scenario provides numerous opportunities for dynamic and flexible human-robot interaction. For example, a variety of communication acts are possible: human generated commands, questions from the robots to the human, etc. Additionally, humans may remotely interact with the robots (e.g., remotely deploy the inspection robot from a habitat) as well as working side-by-side with others (e.g., leading the welder robot to a site ready for welding).

\footnotetext{
${ }^{1}$ Extravehicular Activity (EVA) is work performed by an astronaut outside of a spacecraft.

${ }^{2}$ Intravehicular Activity (IVA) is work performed inside a pressurized module (spacecraft, habitat, etc.
}

\section{B. Data Collection and Baseline}

During the study, we made extensive use of video and audio to record human-robot interaction. Three observers used logging software to code human and robot activity. For each session, we logged time spent moving, time on task, and intervention time (the time a human or robot spent helping another agent). From this data we are able to determine the free time that any of the agents had.

To establish baselines, we collected the time required for each agent to perform primitive tasks: retrieve and assemble panels (astronauts only), move between panels (all), seam weld (astronaut and Robonaut), inspect weld (K10 only). Table I shows the baseline data for the EVA astronauts, K10, and Robonaut. This baseline data can be used to construct time-based workflows of different team configurations.

TABLE I

BASELINE DATA

\begin{tabular}{llc}
\hline \multicolumn{1}{c}{ Agent } & \multicolumn{1}{c}{ Primitive task } & Mean time (sec) \\
\hline \multirow{3}{*}{ EVA astronauts } & move between panels & 3.9 \\
& weld seam & 16.5 \\
& retrieve panel & 13.5 \\
& assemble panel & 7.7 \\
\hline \multirow{2}{*}{ K10 } & inspect seam & 11.3 \\
& move between panels & 12.6 \\
\hline \multirow{2}{*}{ Robonaut } & weld seam & 66.1 \\
& move between panels & 27.4 \\
\hline
\end{tabular}

We should note that it is unlikely that human-robot teams will be able to work as quickly as comparably sized human teams any time soon. But, because cost pressures and other constraints (e.g. payload launch capacity) will keep astronaut teams small, exploration missions will need to include teams of humans and robots. Thus, we are most concerned with identifying task workflows that maximize human-robot team productivity, while reducing the number and duration of EVA's.

\section{Preliminary Results}

One series of tests included seven trials, in which six panels were placed, welded, and inspected by the humanrobot team. The start condition and work sequence in each trial was identical. During these trials, the two EVA astronauts commanded and provided assistance to the robots. The IVA astronaut communicated with the EVA astronauts. IVA also teleoperated and assisted K10 with inspections when needed. Trials 2 to 7 were successfully completed. Figure 3 shows a timeline from Trial 6 , broken down into four activity categories: free time, time on task, time on task with help, and intervention (providing help).

To better understand how the EVA astronauts worked, we computed the percentage of time the astronauts dedicated to each activity category during each trial (Figure 4). The astronauts spent about the same percentage of time on the task during all runs. They spent little time intervening (providing help to robots). Overall, the EVAs had a high percentage of 


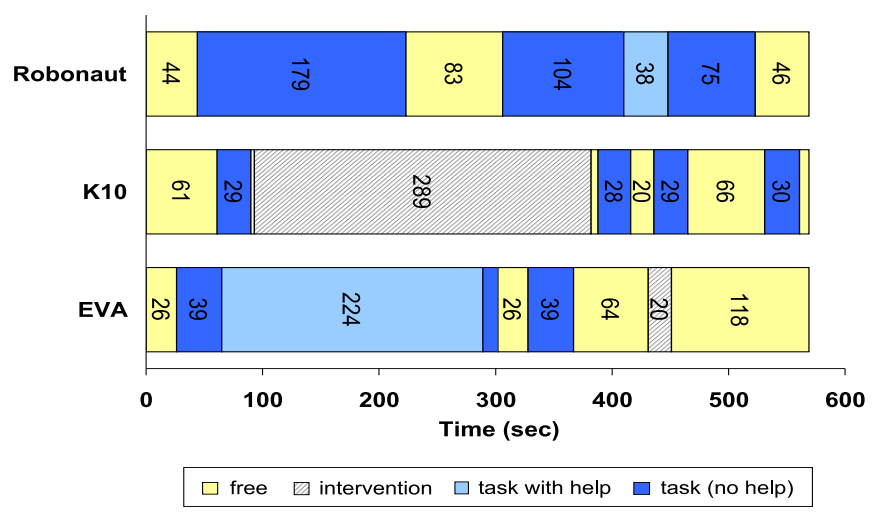

Fig. 3. Activity timeline of human-robot seam welding.

free time, most of which occurred while waiting for robots to finish working at the end of each trial and which would be available for other work.

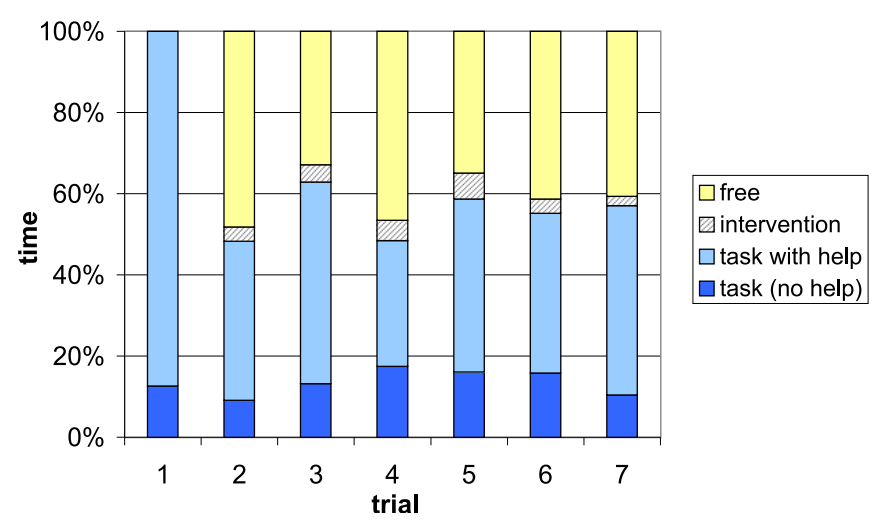

Fig. 4. EVA astronauts time usage

For the IVA we coded four work tasks: monitoring (user interface or line-of-site observation), robot teleoperation, intervention (providing help to robots), and human communication. During all trials, we also asked the IVA to perform a secondary task (reading a newspaper) to better simulate actual work (i.e., so that his attention would not be focused only on the team). Figure 5 shows the percentage of time the IVA spent in each of these activities.

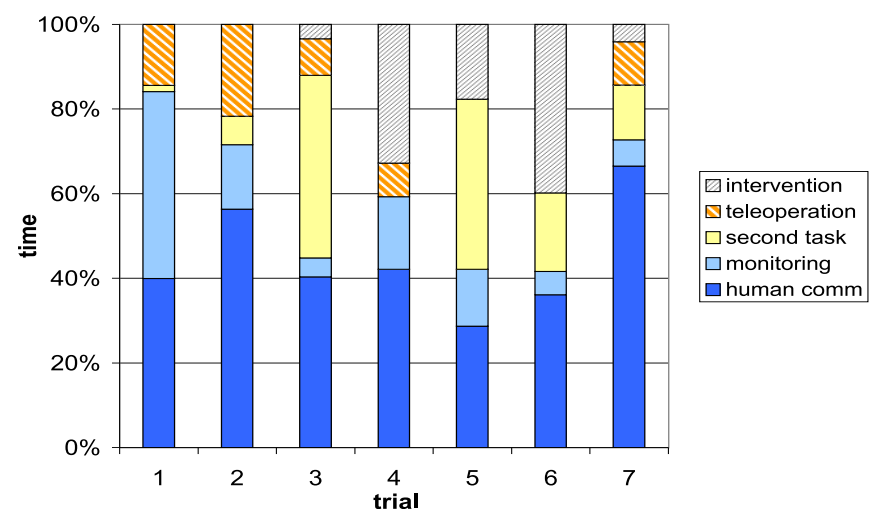

Fig. 5. IVA astronaut time usage
(A)

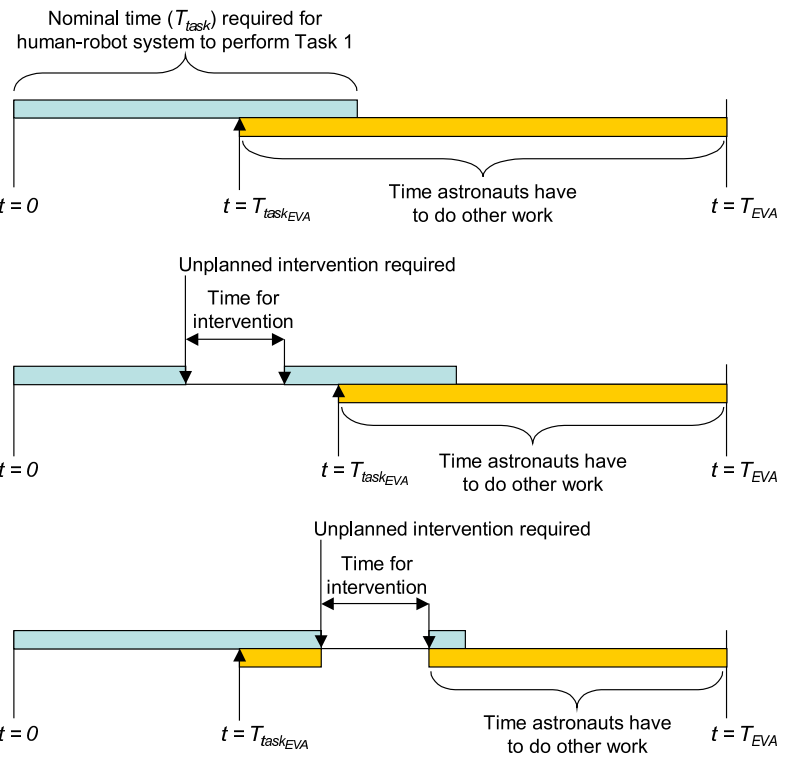

Fig. 6. Effect of interventions on EVA timeline: (a) no interventions, (b) intervention while human and robot working together, (c) intervention after human has started other work.

By far the greatest percentage of the IVA's time was spent on communication. This reflects a need for having the status of the robots and TM more visible to the EVAs. In Trial 7, the IVA was teleoperating K10 while communicating with EVAs but this was not the norm.

\section{INTERVENTION AND EVA}

To better understand how intervention effects EVA, consider the following two measures of EVA productivity:

1) The amount of useful work that astronauts can do.

2) The amount of time astronauts must spend in EVA.

Depending on the nature and the context of the task, it may be important to maximize the first measure (useful work). For other tasks, minimizing the second (exposure) may be the priority. The ideal, of course, is a system that can maximize useful work while minimizing exposure.

Interventions reduce the amount of time the astronauts have available for other work in EVA. Because of the time associated with egress and regress, if robots are "unreliable" and require many interventions, the astronauts may need to stay in EVA and "wait" to handle for these unplanned interventions. Even if the astronauts were to remain out on EVA trying to get other work done, their work would be constantly interrupted by robots requesting intervention. This would directly reduce the productivity of the human-robot team.

Figure 6 illustrates the effects of interventions on different parts of an EVA timeline. A nominal timeline in which the human-robot system performs a collaborative task without unplanned interventions is shown in Figure 6a. The maximum EVA time, $T_{E V A}$, is the maximum amount of time that the human-robot system has to perform a primary task (Task 1). The nominal amount of time for the human-robot system to perform a specific task is labeled $T_{\text {task }}$. The nominal 
amount of time required for the astronauts to perform their part of Task 1 is labeled $T_{\text {task }_{E V A} \text {. The time remaining once }}$ the astronauts complete Task 1 is the time available for them to perform other work.

Interventions that occur while the astronauts are still performing their part of Task 1 increase $T_{t a s k_{E V A}}$ and $T_{\text {task }}$ as shown in Figure 6b. Unplanned interventions after $T_{t a s k_{E V A}}$ lead to a situation in which the astronauts may have to remain in EVA in order to intervene. If the astronauts do not remain in EVA, time penalties associated with egress/regress may be incurred. This situation is shown in Figure 6c. Each of these events significantly increase exposures and decreases the time available for other work.

If, however, the human-robot system is very "reliable" (i.e., robots do not often need help), then astronauts have two choices. On the one hand, when they finish their primary task, they can stay in EVA and begin working on other tasks. This increases the amount of useful work. Alternatively, the astronauts can assume that the robots will complete their tasks without intervention, and thus they can leave EVA. This helps minimize exposure. In other words, a very "reliable" human-robot system provides the option to maximize different productivity measures.

We can examine the effect of intervention on EVA using an analysis inspired by classic reliability techniques [17]. To begin, we first define the following terms:

- Mean Time Between Interventions (MTBI) is the mean time that a human-robot system operates nominally (i.e., outside of intervention). MTBI is analogous to Mean Time Between Failures (MTBF) [18].

- Mean Time to Intervene (MTTI) is the mean time humans and robots spend in intervention. MTTI is analogous to Mean Time To Repair (MTTR) [18].

MTBI is indicative of how often intervention is needed (e.g., how frequently robots ask for help). MTBI is a function of several factors: the environment (and uncertainty in the environment) the system or robot is operating in; the autonomy of the system or robot; and inherent component or system reliabilities.

MTTI is indicative of intervention time (e.g., how long it takes to assist a robot). MTTI is a function of many variables, including (but not limited to): the nature of the problem requiring intervention; the human's expertise with regard to the robot's capabilities and troubleshooting the problem; the amount of information a robot or astronaut is able to gather about the problem; the amount and type of information that can be transferred from robot to human; the distance between human and robot; and communications lag.

\section{A. Time Available for Other Work}

The lifetime of a system is usually considered as having three phases: (1) infant mortality with decreasing failure rate, (2) normal life and (3) wear-out with an increasing failure rate. During normal life, the conditional probability of failure, $\lambda$, given that a component has survived to a given time $t$ is generally constant.

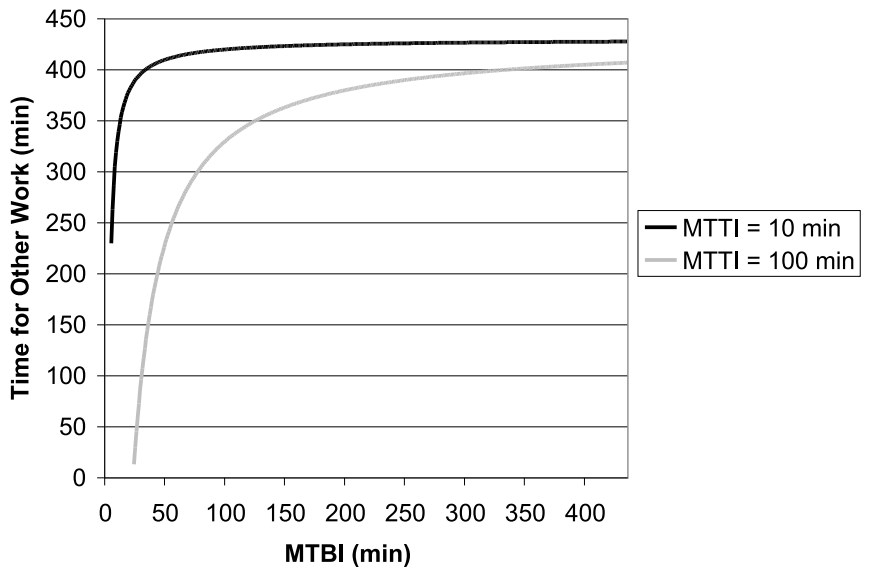

Fig. 7. Time for other work as a function of MTBI and MTTI. EVA values used: $T_{E V A}=8$ hours, $T_{\text {task }}{ }_{E V A}=50 \mathrm{~min}, T_{\text {task }}=100 \mathrm{~min}$.

To examine the influence of MTBI and MTTI on EVA, we will focus on the normal life phase. Assuming that $\lambda$ is constant (i.e. that interventions will occur at a constant rate):

$$
M T B I=\frac{1}{\lambda}=\text { constant }
$$

we can compute the time available for other work using:

$$
T_{\text {other }}=T_{E V A}-T_{\text {task } k_{E A}}-\frac{T_{\text {task }}}{M T B I} M T T I
$$

where $T_{\text {other }}$ is the "time for other work", $T_{E V A}$ is the total EVA time, $T_{t a s k_{E V A}}$ is the nominal time required for astronauts to perform their part of the task, and $T_{\text {task }}$ is the nominal time for the human-robot team to complete the task.

Figure 7 shows how time for other work depends on MTBI, assuming constant MTTI and representative EVA values. Increases in MTTI reduce the time available for human agents to do other work. However as MTBI is increased, the sensitivity to MTTI quickly decreases. Also, increases in MTBI result in increased time available for other work, and the sensitivity to MTBI increases as MTTI increases. This suggests that a designer may be able to compensate for large, or uncertain, MTTI and increase time available to do other work by modestly increasing MTBI.

\section{B. Intervention Probability}

To examine how MTBI and MTTI influence the probability that astronauts will need to intervene, we model the occurrence of interventions using a Poisson distribution. The probability, $F(t)$, that at least one intervention will occur between when the astronauts finish their part of the task and when the human-robot system finishes the task is then:

$$
F(t)=1-\exp \left(-\int_{T_{t a s k_{E V A}, \text { tot }}}^{T_{\text {task }, \text { tot }}} h(s) d s\right)
$$

where $T_{t a s k_{E V A}, t o t}$ is the time required for the astronauts to perform their part of the task (including interventions) and $T_{\text {task,tot }}$ is the total time to perform the task.

Figure 8 shows the probability that intervention is required (after the astronauts have finished their part of the primary 


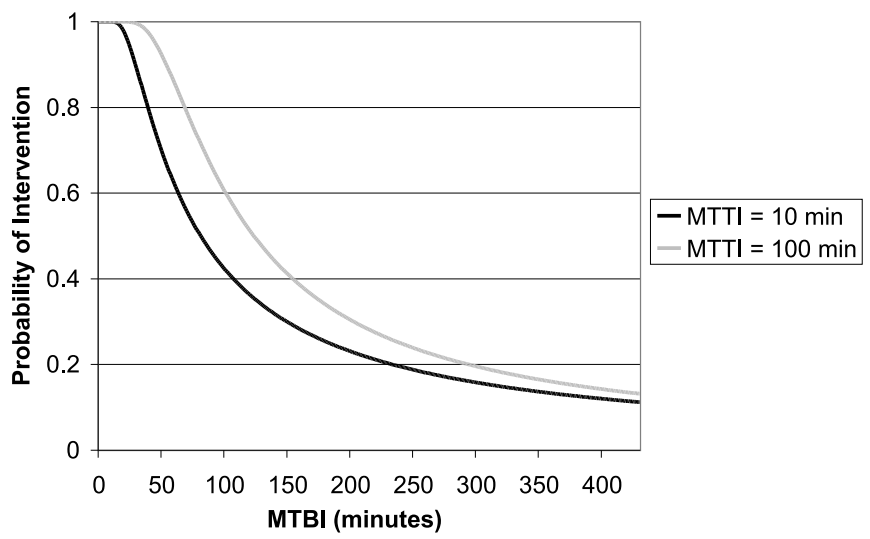

Fig. 8. Probability of Intervention as a function of MTBI and MTTI. EVA values used: $T_{E V A}=8$ hours, $T_{\text {task }_{E V A}}=50 \mathrm{~min}, T_{\text {task }}=100 \mathrm{~min}$.

task) as a function of MTBI, assuming constant MTTI and representative EVA values. Increasing MTTI by an order of magnitude only shifts the curve slightly. Furthermore, as MTBI increases, the effect of changes in MTTI or MTBI decreases. This suggests that uncertain MTTI may not significantly influence the probability of intervention.

\section{FUTURE WORK}

The use case study revealed a number of deficiencies with the current system. In particular, there were a number of times when astronauts used incorrect dialogue. Because the current speech interface does not provide confirmation, the astronauts did not know if a command was received or if the grammar was incorrect. Thus, both the dialogue and the speech interface need to be made more flexible and robust.

We also observed that the astronauts did not always notify the TM when they had completed their work. Although the TM tracks robot progress, a similar facility is needed for humans. One approach would be to add activity monitoring techniques, such as described in [19], into the TM. This would also help ensure that humans are not slowed down by having to inform the system about their activity. In addition, our long-term goal is to extend the HRI/OS to large humanrobot teams. To do this, the TM will need to reason about, and plan for, human and robot resource use.

By far the biggest issue, however, is that of status monitoring. At present, it is difficult for EVA astronauts to assess the status of robots (progress, health, etc.) and software agents. This deficiency impacts both situation awareness and context switching. Although it is possible for the IVA astronaut to provide status reports as an overseer, frequent human-human communications make it difficult for the IVA to accomplish other tasks. Thus, we need to develop status monitoring mechanisms that can function independent of the IVA.

\section{CONCLUSION}

The tools and techniques that the Peer-to-Peer HumanRobot Interaction project is developing have significant potential for improving the human-robot teams. In particular, we believe that software frameworks such as the HRI/OS will enable humans and robots to work more productively together, supporting one another regardless of spatial distribution, communication channel, and user interface.

During the next few years, our goal is to apply peer-topeer HRI to a variety of exploration systems, including the Crew Exploration Vehicle (CEV), lunar landers and rovers. In addition, we plan to study how peer-to-peer HRI can be applied to a range of in-space and surface exploration activities including assembly, inspection, resource mapping, payload transport and site preparation.

\section{ACKNOWLEDGMENT}

We would like to thank Bill Bluethmann, Dan Christian, Larry Edwards, John Hu, Pavithra Rajagopalan, Eli Young, the Ames K10 team and the JSC Robonaut group for their tireless efforts and contributions to this project. This work was sponsored by a grant (HRT-ICP-04-0000-0155) from the NASA Exploration Systems Mission Directorate.

\section{REFERENCES}

[1] NASA, "The vision for space exploration," NASA, Washington, DC, Tech. Rep. NP-2004-01-334-HQ, 2004.

[2] T. Fong, I. Nourbakhsh, et al., "The peer-to-peer human-robot interaction project," in Space 2005, no. AIAA 2005-6750. AIAA, 2005.

[3] A. Steinfeld, T. Fong, et al., "Common metrics for human-robot interaction," in Proc. Conference on Human-Robot Interaction, 2006.

[4] T. Fong, C. Kunz, L. M. Hiatt, and M. Bugajska, "The human-robot interaction operating system," in Proc. Conference on Human-Robot Interaction, 2006.

[5] T. Fong and I. Nourbakhsh, "Interaction challenges in human-robot space exploration," ACM Interactions, vol. 12, no. 2, pp. 42-45, 2005.

[6] A. Cheyer and D. Martin, "The open agent architecture," Journal of Autonomous Agents and Multi-Agent Systems, vol. 4, no. 1, pp. 143148, March 2001.

[7] M. Henning, "A new approach to object-oriented middleware," IEEE Internet Computing, vol. 8, no. 1, 2004.

[8] S. Blisard and M. Skubic, "Modeling spatial referencing language for human-robot interaction," in Proc. IEEE Intl. Workshop on Robot and Human Interactive Communication, 2005.

[9] J. Reitsema, W. Chun, et al., "Team-centered virtual interactive presence for adjustable autonomy," in Space 2005, no. AIAA 2005-6606. AIAA, 2005.

[10] M. Skubic, et al., "Spatial language for human-robot dialogs," IEEE Transactions on Systems, Man and Cybernetics, Part C, vol. 34, no. 2, pp. 154-167, 2004.

[11] N. Cassimatis, J. Trafton, et al., "Integrating cognition, perception, and action through mental simulation in robots," Robotics and Autonomous Systems, vol. 49, no. 1-2, Nov 2004

[12] L. Hiatt, J. Trafton, et al., "A cognitive model for spatial perspective taking," in Proc. 6th Intl. Conference on Cognitive Modelling, 2004.

[13] J. Trafton, N. Cassimatis, et al., "Enabling effective human-robot interaction using perspective-taking in robots," IEEE Trans. on Systems, Man and Cybernetics, Part A, vol. 49, no. 4, July 2005.

[14] S. Johnson and R. Leonard, "Evolution of concepts for lunar bases," in Lunar Bases and Space Activities of the 21st Century, Proc. Lunar and Planetary Institute, 1985, pp. 47-56.

[15] D. Reynerson and C. Reynerson, "Design considerations for remotely operated welding processes," in Proc. Intl. Conference on Robotics and Remote Systems for Hazardous Environments, 2004.

[16] R. Ambrose, H. Aldridge, et al., "Robonaut: Nasa's space humanoid," IEEE Intelligent Systems Journal, Aug 2000.

[17] J. Shah, J. Saleh, and J. Hoffman, "Analytical basis for evaluating the effect of unplanned interventions on the effectiveness of a human-robot system," Reliability Engineering and System Safety, in submission.

[18] IEEE, "Ieee recommended practice for the design of reliable industrial and commercial power systems (gold book)," IEEE, Tech. Rep. IEEE Standard 493-1997, 1998.

[19] R. Collins et al., "A system for video surveillance and monitoring," Robotics Institute, Carnegie Mellon University, Tech. Rep. Technical Report CMU-RI-TR-00-12, 2000. 